\section{(2) OPEN ACCESS}

\title{
TGF $\beta$ promotes low IL10-producing ILC2 with profibrotic ability involved in skin fibrosis in systemic sclerosis
}

\author{
Paôline Laurent, ${ }^{1}$ Benoit Allard (D) , ${ }^{1}$ Pauline Manicki, ${ }^{2}$ Valérie Jolivel, ${ }^{1}$ \\ Emeline Levionnois, ${ }^{1}$ Mohamed Jeljeli, ${ }^{3}$ Pauline Henrot, ${ }^{2}$ Julien Izotte, ${ }^{4}$ \\ Damien Leleu (D) ,' Alexis Groppi, ${ }^{5,6}$ Julien Seneschal, ${ }^{7,8}$ Joel Constans, ${ }^{9}$ \\ Carlo Chizzolini (D) , ${ }^{10}$ Christophe Richez (D) ,,2 Pierre Duffau, ${ }^{1,11}$ Estibaliz Lazaro, ${ }^{1,11}$ \\ Edouard Forcade, 1,12 Thierry Schaeverbeke, ${ }^{1,2}$ Thomas Pradeu, ${ }^{1}$ Frédéric Batteux, ${ }^{3}$ \\ Patrick Blanco, ${ }^{1,13}$ Cécile Contin-Bordes (D) , 1,13 Marie-Elise Truchetet (D) ${ }^{1,2}$
}

\begin{abstract}
Handling editor Josef $S$ Smolen

- Additional supplemental material is published online only. To view, please visit the journal online (http://dx.doi. org/10.1136/annrheumdis2020-219748)
\end{abstract}

For numbered affiliations see end of article.

Correspondence to Pr Marie-Elise Truchetet, Rheumatology, $\mathrm{CHU}$ de

Bordeaux, Bordeaux, Aquitaine, France;

marie-elise.truchetet@chubordeaux.fr

CC-B and M-ET contributed equally.

$\mathrm{PL}$ and $\mathrm{BA}$ are joint first authors.

Received 18 December 2020

Accepted 6 July 2021

Published Online First

20 July 2021

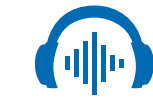

Listen to Podcast ard.bmj.com

Check for updates

(c) Author(s) (or their employer(s)) 2021. Re-use permitted under CC BY-NC. No commercial re-use. See rights and permissions. Published by BMJ.

To cite: Laurent $P$

Allard B, Manicki $P$

et al. Ann Rheum Dis

2021:80:1594-1603.

\section{ABSTRACT}

Objective Innate lymphoid cells-2 (ILC2) were shown to be involved in the development of lung or hepatic fibrosis. We sought to explore the functional and phenotypic heterogeneity of ILC2 in skin fibrosis within systemic sclerosis (SSc).

Methods Blood samples and skin biopsies from healthy donor or patients with SSc were analysed by immunostaining techniques. The fibrotic role of sorted ILC2 was studied in vitro on dermal fibroblast and further explored by transcriptomic approach. Finally, the efficacy of a new treatment against fibrosis was assessed with a mouse model of SSC.

Results We found that ILC2 numbers were increased in the skin of patients with SSC and correlated with the extent of skin fibrosis. In SSc skin, KLRG1 ${ }^{-}$ILC2 (natural ILC2) were dominating over $\mathrm{KLRG} 1^{+} \mathrm{ILC2}$ (inflammatory ILC2). The cytokine transforming growth factor- $\beta$ (TGF $\beta$ ), whose activity is increased in SSc, favoured the expansion of KLRG1' ILC2 simultaneously decreasing their production of interleukin 10 (IL10), which regulates negatively collagen production by dermal fibroblasts. TGF $\beta$-stimulated ILC2 also increased myofibroblast differentiation. Thus, human KLRG1- ILC2 had an enhanced profibrotic activity. In a mouse model of SSc, therapeutic intervention-combining pirfenidone with the administration of IL 10 was required to reduce the numbers of skin infiltrating ILC2, enhancing their expression of KLRG1 and strongly alleviating skin fibrosis.

Conclusion Our results demonstrate a novel role for natural ILC2 and highlight their inter-relationships with TGF $\beta$ and IL10 in the development of skin fibrosis, thereby opening up new therapeutic approaches in SSc.

\section{INTRODUCTION}

Systemic sclerosis (SSc) is a systemic autoimmune disorder characterised by a dysregulated extensive fibrotic process that impacts epithelial barriers, within the gut, lung and skin. ${ }^{1}$ Its pathogenesis remains poorly understood, and treatments for disease progression are limited. While the adaptive immune system has long been considered to be involved in SSc development, recent observations

\section{Key messages}

What is already known about this subject?

- Type 2 innate lymphoid cells (ILC2) have emerged as a player in inflammatory and fibrotic processes.

- Research to date on innate lymphoid cells in systemic sclerosis (SSc) was descriptive suggesting a potential role in the disease development.

- Transforming growth factor- $\beta$ (TGF $\beta$ ) pathway is important in SSc pathophysiology notably through its direct role on fibroblasts.

What does this study add?

- We deeply described ILC2 presence and localisation in fibrotic skin.

- We depicted a new indirect mechanism by which TGF $\beta$ could lead to fibrosis, triggering the switch from an 'inflammatory' phenotype (KLRG1 $\left.{ }^{\text {high }}\right)$ to a 'natural' phenotype (KLRG $1^{\text {low }}$ ) ILC2.

- These TGF $\beta$-activated ILC2, characterised by a diminished interleukin 10 (IL10) production, promote collagen synthesis by fibroblasts.

- Using both in vitro and in vivo models, we established the importance of the combined role of TGF $\beta$ and IL10 in the fibrotic process.

\section{How might this impact on clinical practice or} future developments?

- These data provide important support for the use of combination therapies in SSc.

- The combined use of an antifibrotic drug such as pirfenidone and IL10 could be a new therapeutic approach in this very complicated disease.

have established an important role of the innate immune system. ${ }^{23}$ As an example, a type 2 macrophage signature has been identified in both skin and lung from patients with SSc. ${ }^{4}$

Interestingly, innate lymphoid cells (ILCs) that patrol environmental interfaces to defend against 
infection and protect barrier integrity have emerged as crucial effectors in inflammatory and fibrotic diseases. ${ }^{56}$ Their cytokine production and transcription factor expression allow the identification of three distinct subsets. Type 1 ILC (ILC1) are Tbet expressing cells that produce interferon- $\gamma$ and tumour necrosis factor, and are dependent on IL12 and IL18 for their generation. GATA3 expressing type 2 ILC (ILC2), which are dependent on thymic stromal lymphopoietin (TSLP), IL25 and IL33 release IL5 and IL13 whereas type 3 ILCs (ILC3) express the transcription factor ROR $\gamma$ t, release IL17 and IL22 and are generated after IL23 and IL1 $\beta$ stimulation.

The role of ILC2 was first highlighted in allergic reactions. ${ }^{89}$ More recently, murine studies have shown the role of ILCs in hepatic and pulmonary fibrosis, with a major effect of IL13 production. Interestingly in the context of SSc, TGF $\beta$ is another important profibrotic factor ${ }^{10-12}$ and an essential cytokine for the development of ILC2, ${ }^{13}$ suggesting a potential role of ILC2 on SSc pathogenesis.

To add another level of complexity, ILC2 constitute a heterogeneous population of cells and at least two separate clusters are described based on their differential responses to microenvironment. Inflammatory ILC2 (iILC2) respond to IL25 and produce IL17 in addition to IL13, whereas natural ILC2 (nILC2) respond to IL33 and release high levels of IL13. The differential expression of killer cell lectin-like receptor G1 (KLRG1) has been identified as a marker, with ilLC2 being KLRG1 ${ }^{+}$and nILC2 being KLRG1. iILC2 can be considered a transient progenitor based on its ability to migrate to tissue and then differentiating into nILC2 in response to activation signals. ${ }^{14}{ }^{15}$ However, the relevance of this plasticity in human pathologies remains to be established.

In patients with SSc, we and others have demonstrated increased levels of homeostatic cytokines for ILC2, such as IL25, IL33 and TSLP. ${ }^{16-18}$ Furthermore, our group found that TSLP is increased in the blood and skin of patients with SSc, with levels correlating to skin fibrosis. ${ }^{18}$ In human SSc, the role of ILC2 remains elusive as only one observational study showed an increased proportion of circulating ILC2 at the blood and tissue level, ${ }^{19}$ thus questioning the potential implication of ILC2 in the fibrotic process.

In our study, we demonstrate the potential role of ILC2 in the establishment of fibrosis in human SSc. We showed that KLRG1 expression on ILC2 was linked to the fibrotic stage of the disease. Mechanistically, in vitro and in vivo studies revealed that this switch operates in a TGF $\beta$-dependent manner, leading to a decrease in IL10 production and a profibrotic phenotype. Interestingly, while pirfenidone alone (acting partly by the inhibition of TGF $\beta$-induced effects) failed to significantly affect the fibrotic process in vivo, the addition of IL10 synergistically alleviated fibrosis. Overall, this study unravels a new role for ILC2 in fibrotic diseases and paves the way for new therapeutic strategies for human SSc.

\section{METHODS}

Materials and methods are described in the online supplemental file.

\section{RESULTS}

\section{Number of circulating ILC2 is decreased in human SSc with the extent of skin fibrosis}

To investigate the potential contribution of ILC2 in SSc pathogenesis, we first monitored the total ILC population and subpopulations in the whole blood of patients with SSc (SSc, n=73) and age-matched and sex-matched healthy donors (HDs) $(\mathrm{n}=59)$ (table 1).

The gating strategy to identify ILCs in the peripheral blood is described in the online supplemental figure 1A. The frequency and absolute numbers of ILCs (defined as Lin $\mathrm{CD} 45^{+} \mathrm{CD} 127^{+}$, figure $\left.1 \mathrm{~A}\right)$ were lower in SSc compared with HDs $(0.04 \% \pm 0.02 \%$ vs $0.09 \% \pm 0.07 \%, \mathrm{p}<0.0001$ and $0.0009 \pm 0.0003$ vs $0.002 \pm 0.001, p=0.0004$; figure $1 \mathrm{~B}$ and online supplemental figure 1AC, respectively), with ILC1, ILC2 and ILC3 being 74\%, $15 \%$ and $11 \%$ in SSc and $55 \%, 18 \%$ and $27 \%$ in HDs, respectively (figure 1C). When focusing on ILC2 defined as $\mathrm{Lin}^{-} \mathrm{CD} 45^{+} \mathrm{CD} 127^{+} \mathrm{CRTH} 2^{+}$, their frequency and absolute numbers were approximately three times lower in SSc

\begin{tabular}{|c|c|c|c|c|}
\hline & Patients with IcSSc $(n=50)$ & Patients with dcSSc $(n=23)$ & All patients with SSc ( $n=73)$ & $P$ value* \\
\hline Female (\%) & $39(78)$ & $9(40)$ & $48(65.8)$ & \\
\hline Age at onset, mean \pm SD years $\dagger$ & $48.7 \pm 13.2$ & $49.9 \pm 14.5$ & $49 \pm 13.5$ & ns \\
\hline Disease duration, mean $\pm S D$ years $t$ & $10.7 \pm 6.0$ & $11.9 \pm 9.47$ & $11.5 \pm 8.6$ & ns \\
\hline $\operatorname{RP}(\%)$ & $50(100)$ & $23(100)$ & $73(100)$ & ns \\
\hline Digital ulcers (\%) & $20(40)$ & $7(30.8)$ & $27(37)$ & 0.02 \\
\hline mRSS, mean \pm SD & $5.9 \pm 5.5$ & $24.6 \pm 12.7$ & $11.1 \pm 11.6 \neq$ & $<0.0001$ \\
\hline PAH $(\%)$ & $7(14)$ & $1(4.3)$ & $8(11) \S$ & ns \\
\hline Interstitial lung disease (\%) & $11(22)$ & $13(56.5)$ & $24(32.9) \uparrow$ & ns \\
\hline Lung fibrosis (\%) & $7(14)$ & $1(4.3)$ & $8(11) 9$ & ns \\
\hline Renal crisis (\%) & $1(2)$ & $0(0)$ & $1(1.4)^{\pi}$ & ns \\
\hline Antinuclear autoantibody-positive & $50(100)$ & $23(100)$ & $73(100)$ & ns \\
\hline Anticentromere antibody-positive & $25(50)$ & $1(4.34)$ & $26(35.6)$ & ns \\
\hline Antitopoisomerase antibody-positive & $4(8)$ & $11(47.8)$ & $15(20.5)$ & ns \\
\hline Anti-ARNIII polymerase antibody-positive & $1(2)$ & $1(4.34)$ & $2(2.7)$ & ns \\
\hline Immunomodulatory agents & $44(22)$ & $60.8(14)$ & $49.3(36)$ & ns \\
\hline
\end{tabular}

Except where indicated otherwise, values are the number (\%).

*I ICSSc versus dcSSc.

tAge at onset of symptoms other than RP and disease duration since symptoms other than RP.

$\ddagger$ Data were available for 34 patients.

$\S$ Data were available for 36 patients.

IData were available for 35 patients.

dcSSc, diffuse cutaneous SSc; ICSSc, limited cutaneous systemic sclerosis; mRSS, modified Rodnan skin thickness score; ns, not significant; PAH, pulmonary hypertension; RP,

Raynaud's phenomenon; SSc, systemic sclerosis. 
A

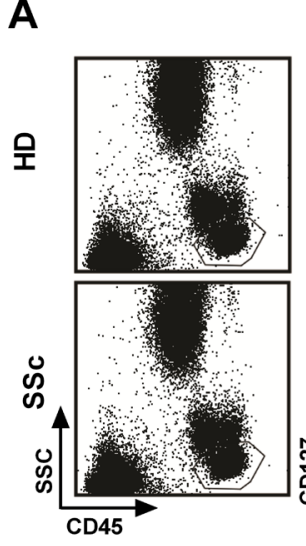

Gated on CD45
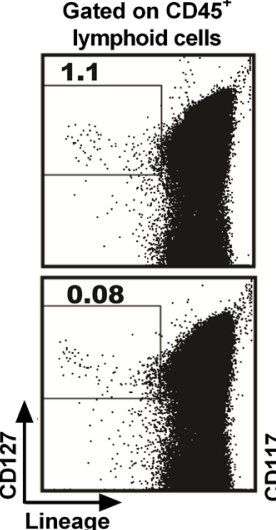

Gated on
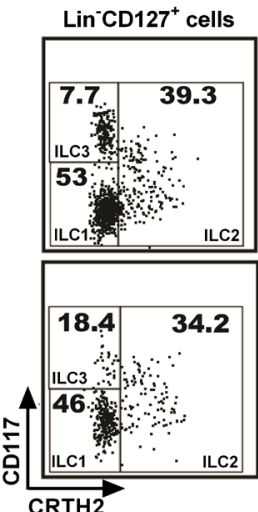

D

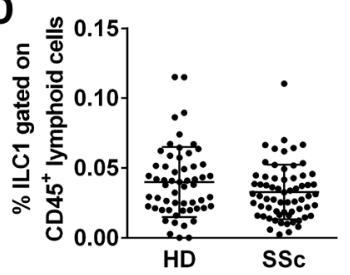

E

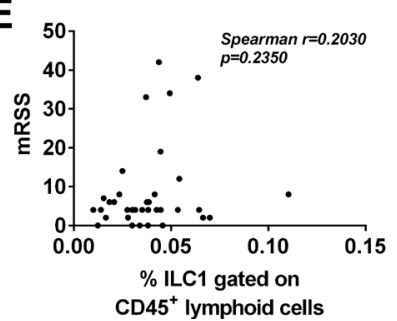

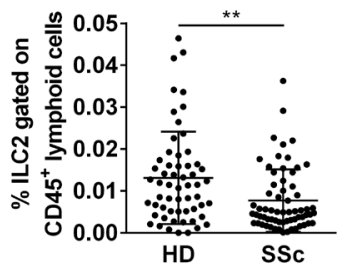

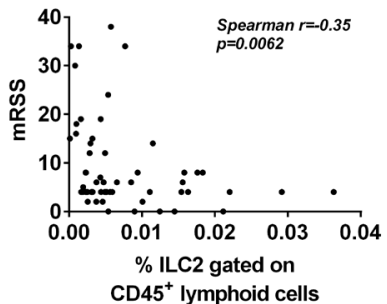

B

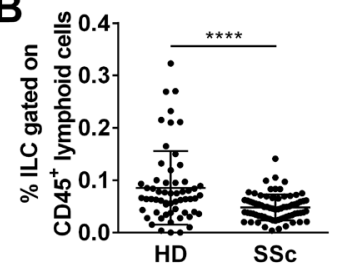

C
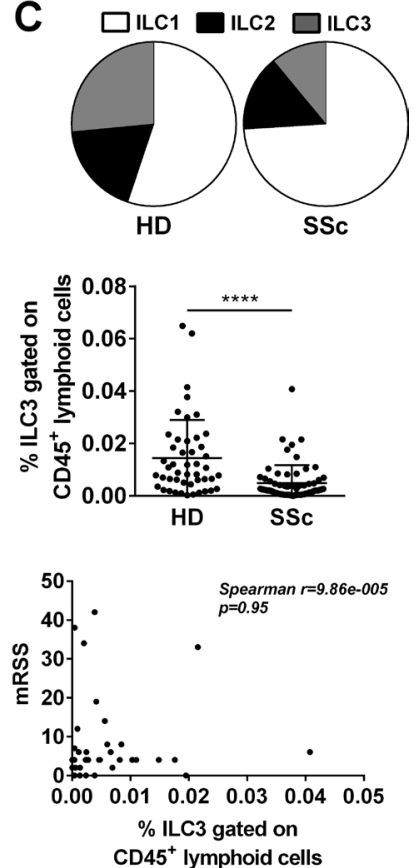

Figure 1 Characterisation of innate lymphoid cells (ILCs) in the blood of patients with systemic sclerosis (SSC) and healthy donors (HDs). (A) Representative dot plot of circulating ILCS in the HD and SSC blood samples and (B) ILC frequency quantification. (C) Proportion of ILC subsets in the blood from HDs and patients with SSc. (D) Percentage of circulating ILC1, ILC2 and ILC3 in the HD and SSC blood. (E) Correlations between circulating ILC1, ILC2 and ILC3 with the extent of cutaneous fibrosis (modified Rodnan skin thickness score (mRSS)). Data are the mean \pm SEM $(n=59$ and 73 for HDs and patients with SSC, respectively). Comparisons between groups were calculated using Mann-Whitney U test. ${ }^{*} \mathrm{P}<0.01 ;{ }^{* * * *} \mathrm{p}<0.0001$.

compared with HDs (figure 1D and online supplemental figure $1 \mathrm{BD}$, respectively). However, no differences in the frequency of $\mathrm{KLRG}^{+}$ILC2 were found (online supplemental figure 1CE). Decreased were also the frequency and absolute numbers of ILC3, but not of ILC1 when SSc and HDs were compared (figure 1D and online supplemental figure 1BD).

To evaluate the clinical relevance of these observations, we analysed the ILC numbers to different relevant clinical parameters. We specifically observed a correlation between the quantity of circulating ILC2 and the modified Rodnan skin thickness score (mRSS), showing that lower is the amount of circulating ILC2 and higher is the cutaneous fibrosis (figure $1 \mathrm{E}$ and online supplemental figure 1DF). Of note, the ILC1 and ILC3 frequencies were not correlated with the mRSS. When comparing patients with or without interstitial lung disease, we found no difference on the $\%$ and the absolute count of ILC2 in the whole blood and there were also no differences according to the severity of interstitial lung disease or the disease duration (data not shown). Collectively, our data indicate that patients with SSc are characterised by a significant reduction in the proportion and number of circulating ILC2, which is correlated with the extent of skin fibrosis.

\section{ILC2 are increased in human SSC skin and correlated with the extent of fibrosis}

Since circulating ILCs were decreased, we further characterise ILCs infiltration in the skin. We first extracted cells from the skin and performed flow cytometry analysis. Representative staining depicting the gating strategy for ILCs and subpopulation categorisation is shown in figure $2 \mathrm{~A}$ and online supplemental figure $2 \mathrm{~A}$. As suspected, we found that the percentage of total ILCs among $\mathrm{CD} 45^{+}$cells was increased in the SSc skin compared with that in the HD skin (figure 2B). When evaluating the repartition of ILC subsets among the total skin ILCs, we observed that $69 \%$ were ILC2 in the HDs vs $77 \%$ in the patients with SSc, $27 \%$ were ILC1 in the HDs vs $19 \%$ in the patients with SSc (figure 2C), while ILC3 were barely detectable. When analysed among CD $45^{+}$cells, a significant increase in ILC2 was observed in the SSc skin compared with the HD skin (figure 2D). The ILC1 frequency was similar in the patients with SSc and HDs (online supplemental figure $2 \mathrm{AB}$ ), even if the proportion among ILCs was decreased in the patients with SSc compared with that in the HDs (figure 2C). The increased percentage of ILC2s among $\mathrm{CD} 45^{+}$skin cells was correlated with the extent of skin fibrosis (figure 2E), whereas the ILC1 percentage did not show this correlation (online supplemental figure 2BC).

To validate this analysis and gain more insights on the precise localisation and quantification of ILC2 in the skin, we performed tissue immunofluorescence staining. Representative staining of ILC2 $\left(\mathrm{Lin}^{-} \mathrm{DAPI}^{+} \mathrm{CRTH}_{2}{ }^{+}\right.$) for an HD and an SSc patient is depicted in figure $2 \mathrm{H}$,I. An analysis of the ILC2 distribution and quantification revealed that the number of ILC2 per surface area $\left(\mathrm{mm}^{2}\right)$ and the percentage of ILC2 (per total cell count) were increased in the patients with SSc compared with the HDs in the dermis (figure 2F 
A
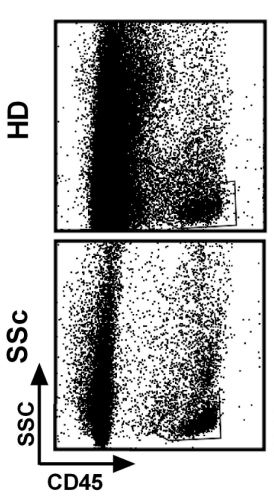

D

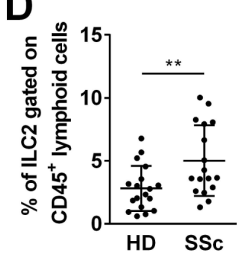

E
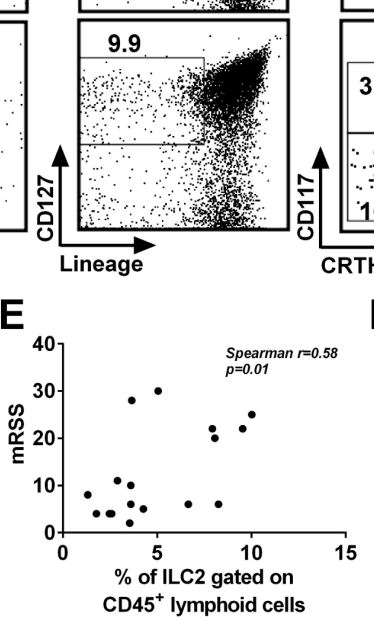
Gated on CD45
Gated on lymphoid cells

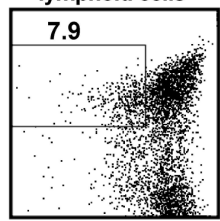

Lin $^{-C D} 127^{+}$cells
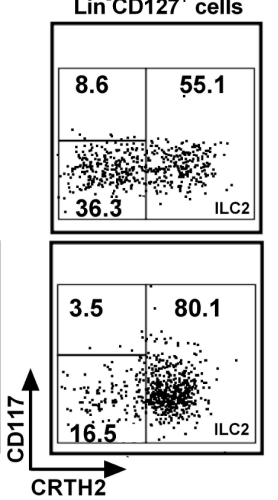

$F$

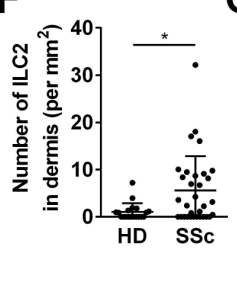

B

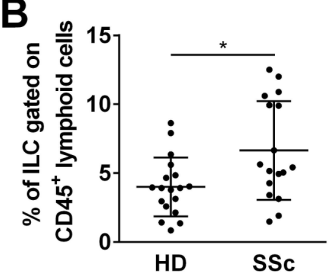

C

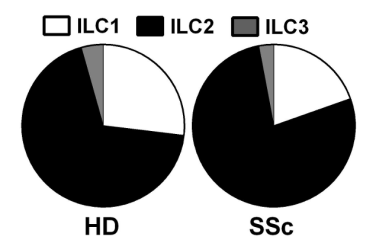

G

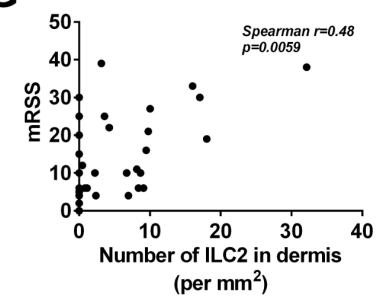

H

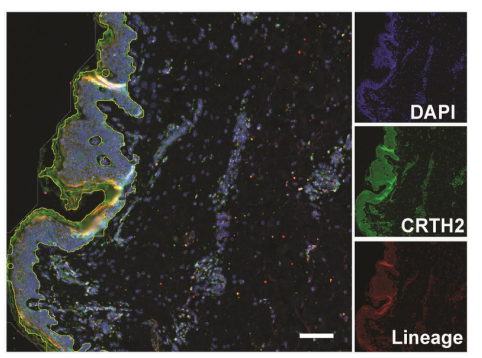

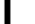

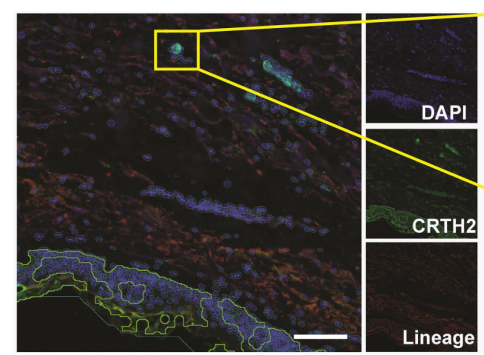

Figure 2 Characterisation of cutaneous innate lymphoid cells (ILC)-2 (ILC2) in patients with systemic sclerosis (SSc) and healthy donors (HDs). (A) Representative dot plot of cutaneous ILCs in the HD and SSc skin samples and (B) ILC frequency quantification. (C) Proportion of ILC subsets in the skin of HDs and patients with SSC. (D) Percentage of ILC2 among CD45 lymphoid cells in the HD and SSc skin samples. (E) Positive correlation between the percentage of ILC2 among CD45 ${ }^{+}$cells and the extent of cutaneous fibrosis (modified Rodnan skin thickness score (mRSS)). (F) Number of ILC2 per $\mathrm{mm}^{2}$ in the dermis of HD and SSc skin. (G) Correlation between the extent of cutaneous fibrosis (mRSS) and the number of ILC2 per mm ${ }^{2}$ in the dermis. ( $\mathrm{H}$ and $\mathrm{I})$ Representative picture of an immunofluorescence assay using anti-CRTH2-based immunofluorescence (green) and antilineage (CD3, CD11b and FceR1)-based immunofluorescence (red) performed to detect CRTH2 ${ }^{+}$Lin $^{-}$ILC2 in the HD and SSC skin samples (scale bars=100 $\mu \mathrm{m}$ ). Bar graphs show data as the mean \pm SEM ( $n=18-20$ and 17-32 for HD and SSc, respectively). Comparisons between groups were calculated using Mann-Whitney U test. ${ }^{*} \mathrm{P}<0.05 ;{ }^{*} \mathrm{p}<0.01$.

and online supplemental figure 2CD). Of interest, the number of ILC2 per $\mathrm{mm}^{2}$ in the dermis was positively correlated with the extent of skin fibrosis as assessed by mRSS at the time of biopsy (figure 2G).

Overall, these results show that ILC2 are increased in human SSc skin and their frequency in the dermis is associated with skin fibrosis.

\section{KLRG1 expression on skin ILC2 is modified over the course of the disease}

To determine the phenotype of cutaneous ILC2 in the SSc skin, we studied the expression of several markers: HLA DR, OX40L, CCR10, CCR6, CLA, TSLPR and KLRG1 (figure 3A,B). The percentage of ILC2 expressing HLA-DR, OX40L, CCR6, CCR10, CLA and TSLPR was similar in the skin of the patients with SSc and HDs. In sharp contrast, the percentage of KLRG1positive cells was significantly lower in the SSc skin (figure 3A,B). Interestingly, the percentage of ILC2 $\mathrm{KLRG}^{+}$tends to decrease with the extent of cutaneous fibrosis (figure 3C).
Altogether, these results indicated that surface KLRG1 expression on ILC2 decreases in the SSc skin and may correlate with the extent of skin fibrosis thus raising the question of the functional relevance of this observation.

\section{TGF $\beta$ promotes in vitro KLRG1 modulation of ILC2 and impacts IL10 secretion}

The relevance of KLRG1 in ILC2 modulation are uncertain and remains to be proven, particularly in human SSc. To identify the factors implicated in KLRG1 modulation, we sorted ILC2 from the peripheral blood of HDs (online supplemental figure $3 \mathrm{~A}$ ) and expanded this population in vitro in the presence of IL1 $\beta$ and IL 2 as previously described. ${ }^{20}$ At the end of the culture, ILC2 were consistently $\mathrm{CD} 127^{+} \mathrm{CRTH}_{2}{ }^{+}$(online supplemental figure $3 \mathrm{~B}$ ) and characterised by high expression of GATA3 (online supplemental figure $3 \mathrm{C}) .{ }^{21}$ We then tested cytokines, specifically involved in human SSc, for their ability to modulate KLRG1 expression on expanded ILC2. IL33, IL4, TSLP and IL25 did not modify KLRG1 expression while 


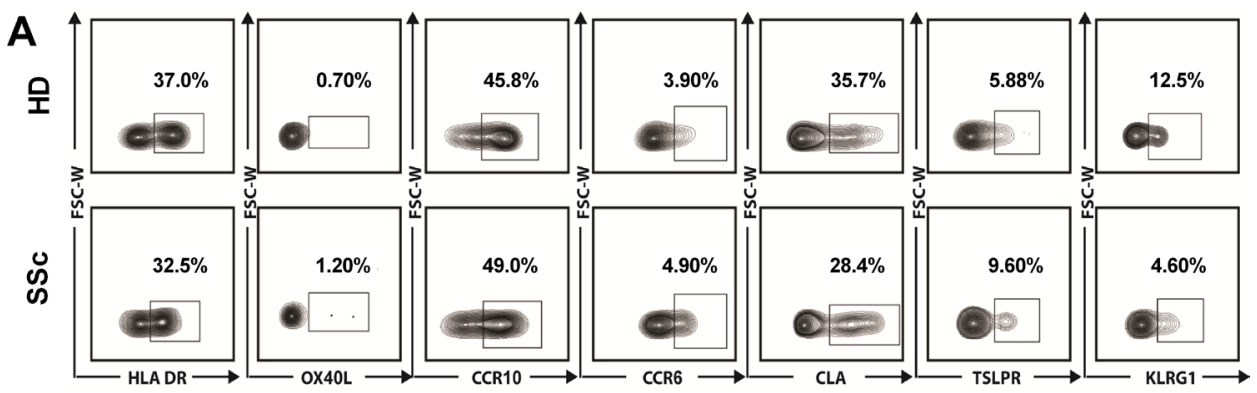

B
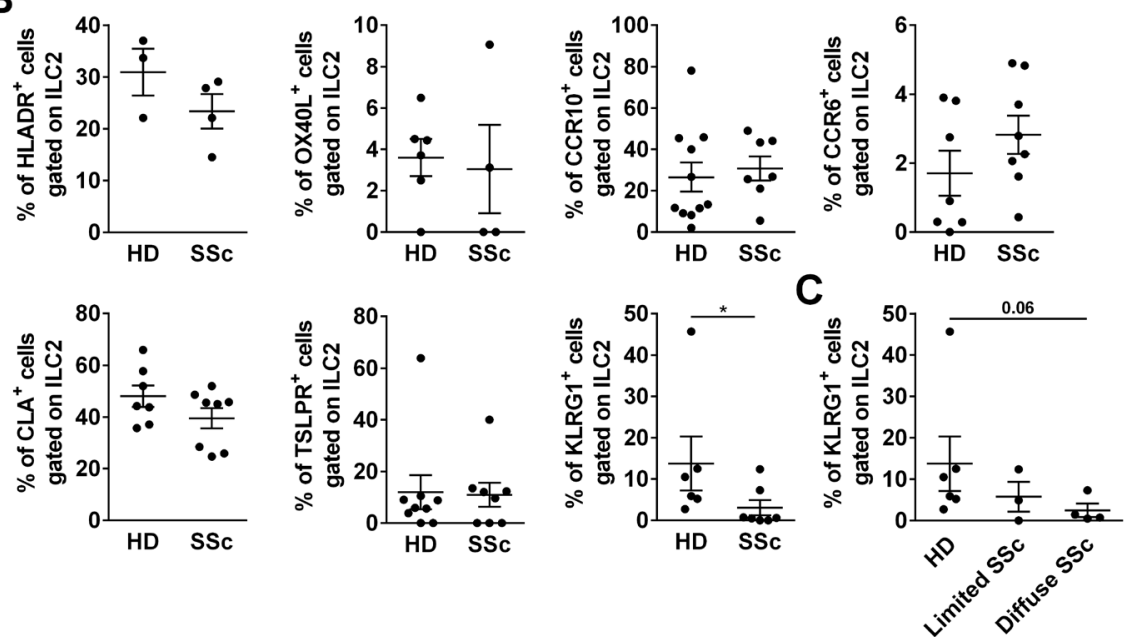

Figure 3 Decrease of killer cell lectin-like receptor G1 (KLRG1) expression on innate lymphoid cells (ILC)-2 (ILC2) in the skin of patients with systemic sclerosis (SSC). (A and B) Expression of HLA DR, OX40L, CCR10, CCR6, CLA, TSLPR and KLRG1 on skin ILC2 in the healthy donors (HDs) and patients with SSc. (C) Percentage of KLRG1 ${ }^{+}$cells among ILC2s in patients with SSc with limited (Rodnan score $<10$ ) and diffuse (Rodnan score $>10$ ) in patients with SSC. Bar graphs show data as the mean \pm SEM ( $n=3-11$ and 4-8 for HD and SSC, respectively). Comparisons between groups were calculated using Mann-Whitney $\mathrm{U}$ test. ${ }^{*} \mathrm{P}<0.05$.

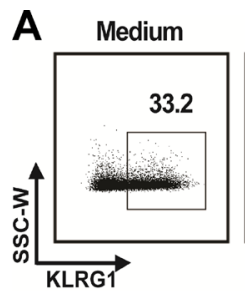

B

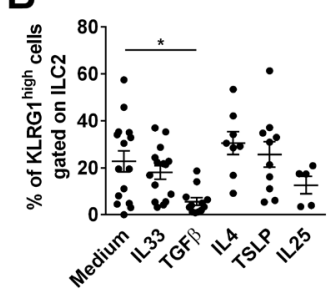

IL33

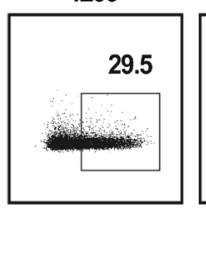

C

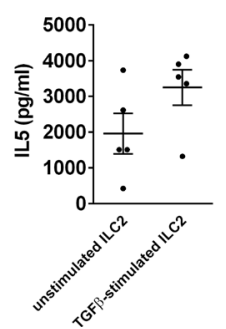

TGF $\beta$

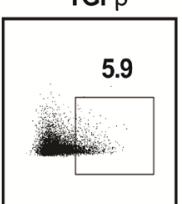

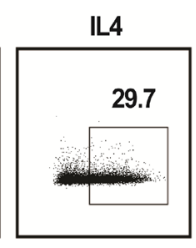
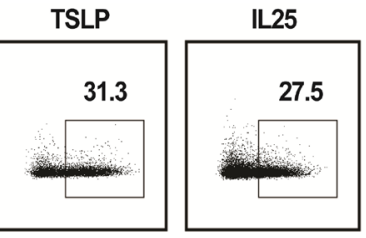

Figure 4 Transforming growth factor- $\beta$ (TGF $\beta$ ) affects the innate lymphoid cells (ILC)-2 (ILC2) phenotype by decreasing killer cell lectin-like receptor G1 (KLRG1) expression and interleukin (IL)10 production. (A) Representative dot blot of KLRG1 expression and (B) percentage of KLRG1 ${ }^{+}$cells in unstimulated ILC2 (medium) and ILC2 stimulated with IL33, TGF $\beta$, IL4, thymic stromal lymphopoietin (TSLP) and IL25 after 20 days of amplification. (C) Secretion of IL5, IL9, IL13 and IL10 assessed by a cytokine bead assay (CBA) in both types of ILC2 supernatants. Bar graphs show data as the mean \pm SEM ( $n=5-16$ for KLRG1 expression and $n=4-7$ for CBA). Comparisons between groups were calculated using Kruskal-Wallis or Wilcoxon tests. ${ }^{*} \mathrm{P}<0.05$.

TGF $\beta$ dramatically decreased the expression of KLRG1 on ILC2 (figure 4A,B).

We then evaluated the functional consequences of TGF $\beta$ exposure on ILC2 by analysing its impact on cytokine production. The levels of IL5, IL9 and IL13 were similar in ILC2 exposed or not to TGF 3 (figure 4C). In contrast, the production of IL10 in TGF 3 -primed ILC2 was significantly reduced compared with that of controls.

Overall, these data indicate that TGF $\beta$ downregulates KLRG1 expression on ILC2, whose phenotype is associated with a decreased capability to produce IL10. 
IL10 decreased production by TGF $\beta$-stimulated ILC2 leads to a profibrotic profile by fibroblasts

Our observations suggest that the KLRG1- ILC2 population could be involved in the fibrotic process. We therefore investigated the ability of TGF $\beta$-stimulated ILC2 to modify the fibrotic response. To this end, supernatants (SN) from TGF 3 -stimulated or unstimulated ILC2 were added to dermal fibroblasts. After 1 day of co-culture, the expression of type I collagen (COL1A1) and matrix metalloproteinase-1 (MMP-1) was evaluated. As expected, TGF $\beta$ alone increased COL1A1 and decreased MMP1 messenger RNA (mRNA) expression (figure 5A). TGF $\beta$ neutralisation completely blocked these changes. Interestingly, SN from TGF $\beta$-stimulated ILC2 increased the COL1A1 mRNA expression while the SN from unstimulated ILC2 did not affect the COL1A1 mRNA levels. Importantly, TGF $\beta$ neutralisation in TGF $\beta$-stimulated ILC2 did not affect these results, thereby ruling out the effect of the initial exogenously added TGF $\beta$ on the induction of COL1A1 mRNA in fibroblasts. Furthermore, ILC2 SN dramatically increased the MMP1 mRNA expression independently of the priming conditions (figure $5 \mathrm{~A}$ ). The profibrotic activity of TGF $\beta$-stimulated ILC2 was emphasised by the COL1A1/MMP1 ratio as a surrogate of collagen turnover, which was increased, suggesting an enhanced collagen deposition over degradation (figure $5 \mathrm{~B}$ ). While no difference of expression was observed for COL1A2, the SN of TGF $\beta$-stimulated ILC2 also increased the mRNA of fibronectin (figure 5C).

Since the production of IL10 was specifically reduced in TGF $\beta$-stimulated ILC2, we next evaluated the role of IL10 on their profibrotic activity. Of interest, when IL10 was neutralised in control ILC2 SN we observed enhanced COL1A1 mRNA expression of dermal fibroblast (figure 5D). Conversely, the addition of IL10 to TGF $\beta$-stimulated ILC2 SN led to a dramatic decrease in COL1A1 mRNA expression. We then confirmed these results at the protein level (figure 5E). To further explore the fibroblast activation, we measured the proliferation and the differentiation into myofibroblasts. While no difference was observed regarding the proliferation (figure 5F), we found a significant increase expression of $\alpha$-smooth muscle actin when fibroblasts were incubated with the SN of TGF $\beta$-stimulated ILC2, independently of IL10 (figure 5G). As previously, we paid attention to incubate the $\mathrm{SN}$ of TGF $\beta$-stimulated ILC2 with antiTGF $\beta$ blocking antibody, excluding the possibility that residual TGF $\beta$ from ILC 2 activation may have mediated myofibroblast differentiation.

Collectively, our data show that TGF $\beta$ favours the generation of KLRG1 ILC2 characterised by low IL10 production capacity, which simultaneously results in their enhanced profibrotic capacity.

\section{Transcriptomic analysis reveals additional fibrotic potential of TGF $\beta$-stimulated ILC2}

To look further on the fibrotic potential of human ILC2, we evaluated the transcriptome of TGF $\beta$-stimulated ILC 2 compared with unstimulated ILC2 by RNAseq (online supplemental figure $4 \mathrm{~A})$. The heat map shows modification in gene expression of ILC2 triggered by TGF $\beta$ activation, with 2840 genes being differentially expressed (p-adjusted value $<0.01$ ). The enrichment analysis with R library Gprofiler2 (V.0.2.0), using Gene Ontology databases, indicated SMADs activation ('heteromeric SMAD protein complex'; GO:0071144; p adjusted value $=4.01 \mathrm{E}^{-2}$ and 'SMAD protein complex'; GO:0071141; $\mathrm{p}$ adjusted value $=4.45 \mathrm{E}^{-2}$ ) and immune regulation (online supplemental table E3, E4, E5, E6 and E7). In the first 60 most upregulated genes (online supplemental table E8), we look at
A
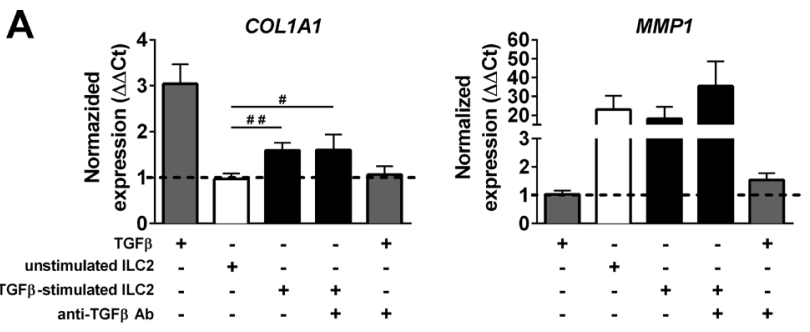

B

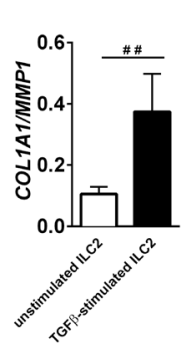

C COLAA2
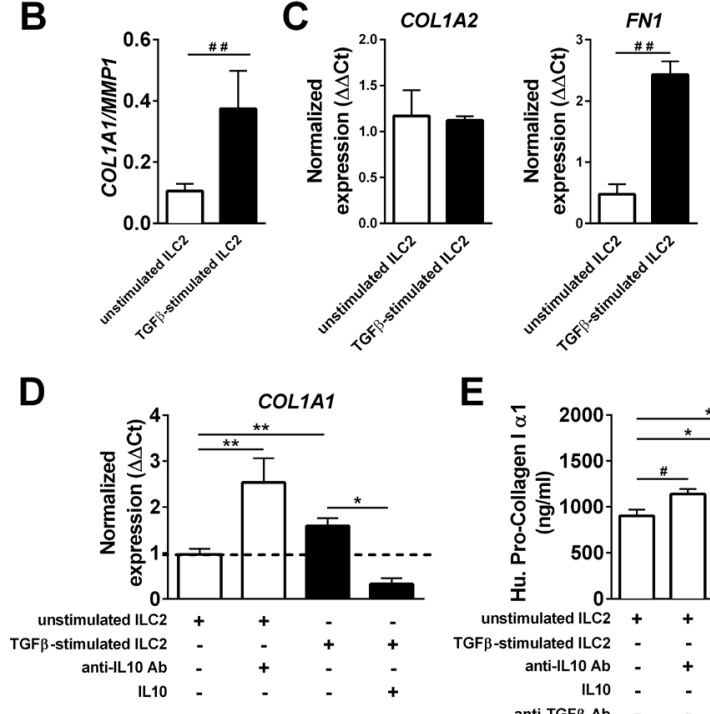

E

$\mathbf{F}$

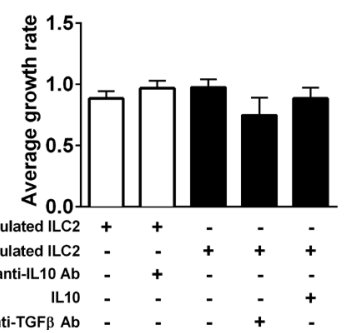

G

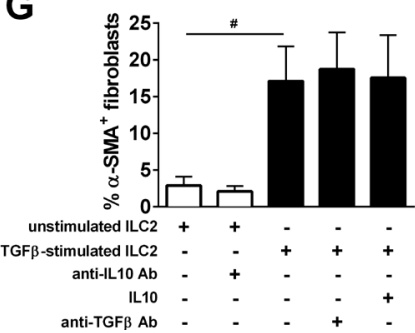

Figure 5 Transforming growth factor- $\beta$ (TGF $\beta$ ) exerts an indirect fibrotic role through innate lymphoid cells (ILC)-2 (ILC2) and interleukin (IL)10 secretion. (A) Q-RT-PCR analysis of type I collagen (COL1A1) and matrix metalloproteinase-1 (MMP-1) messenger RNA (mRNA) expression and (B) COL1A1/MMP1 ratio, in fibroblasts from healthy donors (HDs), incubated with TGF $\beta$ or supernatant of unstimulated ILC2 or supernatant of TGF $\beta$-stimulated ILC2 with or without blocking antibodies against TGF $\beta$. (C) Q-RT-PCR analysis of COL1A2 and FN1 mRNA expression. (D) Q-RT-PCR analysis of COL1A1 expression in fibroblasts incubated with supernatant of unstimulated ILC2 (in the presence or absence of anti-IL10 antibodies) and supernatant of TGF $\beta$ stimulated ILC2 (with or without IL10). (E) Human pro-collagen I $\alpha 1$ concentration were analysed in the supernatants of fibroblasts from HDs after 48 hours of activation with ILC2 supernatants. (F) Average growth rate of fibroblasts were analysed after 48 hours of proliferation. (G) The percentage of $\alpha$-smooth muscle actin positive fibroblasts were analysed after 72 hours of activation. Bar graphs show data as the mean \pm SEM $(n=7-15)$. Comparisons between groups were calculated using the paired Wilcoxon (\#) or the Kruskal-Wallis tests $\left({ }^{*}\right) .{ }^{*} \mathrm{P}<0.05$; ${ }^{* *} \mathrm{p}<0.01$.

genes already described in fibrosis mechanisms. Interestingly, we observed an upregulation of LTC4S (leukotriene C4 synthase) $\left(\log 2\right.$ fold change $=2.71 ; \mathrm{p}$ adjusted value $\left.=9.89 \mathrm{E}^{-22}\right)$. Regarding downregulated genes, we found a decrease of IL10 (log2 fold 
A

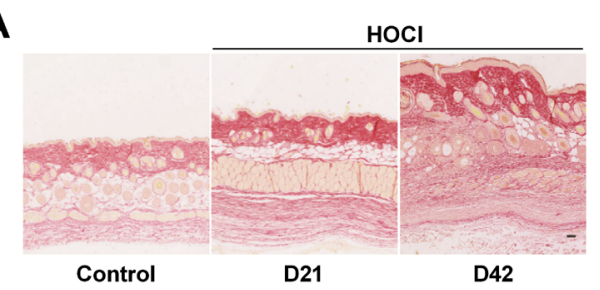

B

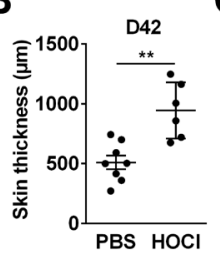

C

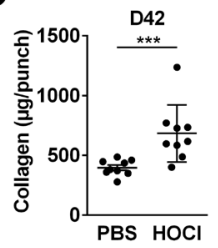

E

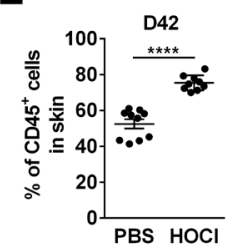

Gated on ILC

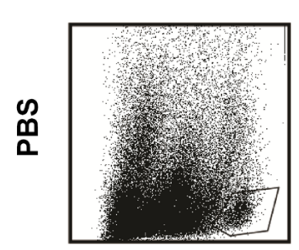

Gated on $\mathrm{CD}^{+} 5^{+}$
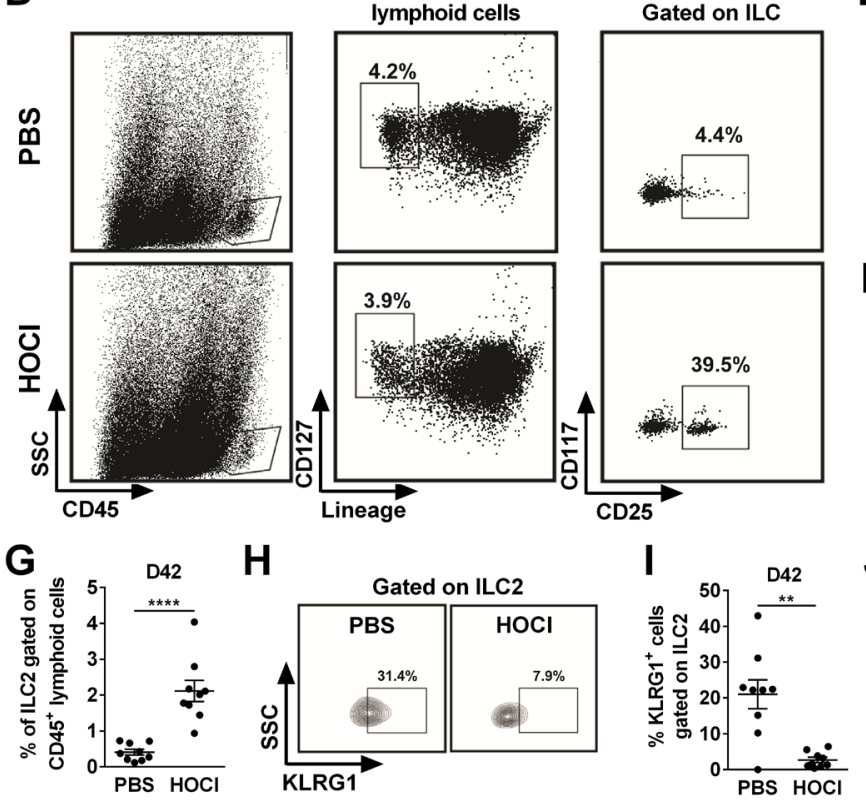

$\mathbf{F}$
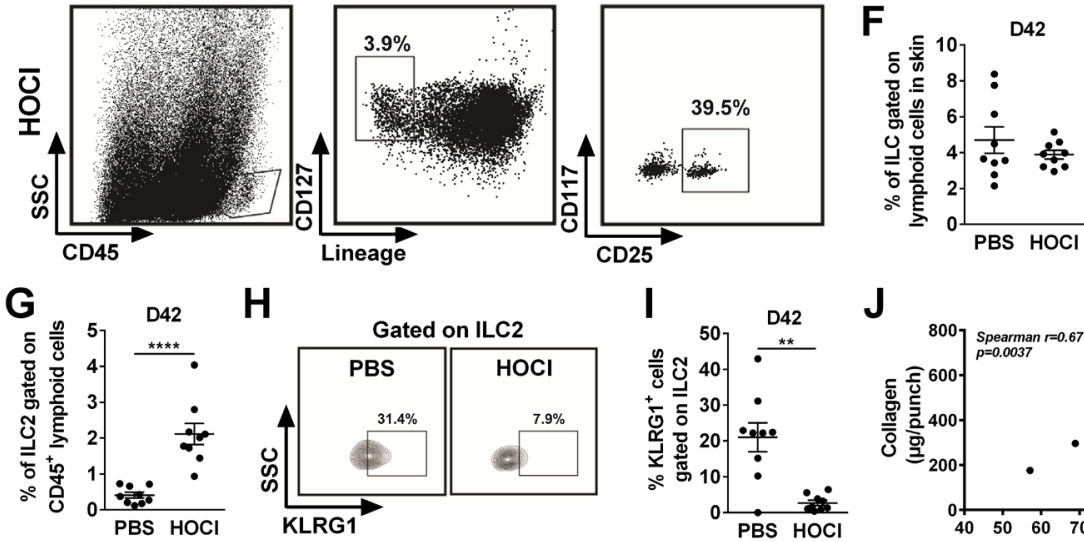

H

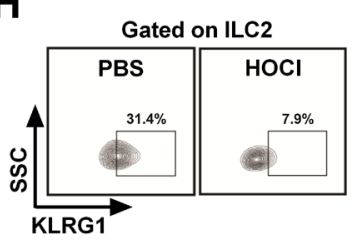

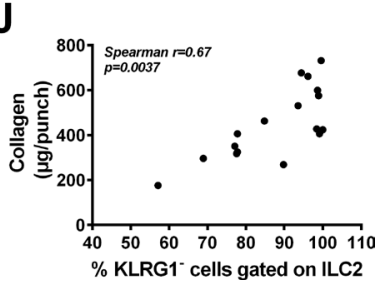

Figure 6 Characterisation of innate lymphoid cells (ILC)-2 (ILC2) in the skin of phosphate-buffered saline (PBS)-treated and hypochlorous acid ( $\mathrm{HOCl}$ )-treated mice. (A) Picrosirius red staining of the control and systemic sclerosis (SSc) skin mice at day 42 (scale bar=10 $\mu \mathrm{m}$ ). (B and C) Measure of skin thickness $(\mu \mathrm{m})$ and collagen $(\mu \mathrm{g})$ in the skin of PBS-treated and $\mathrm{HOCl}$-treated mice at day 42 . (D) Representative dot plot of cutaneous ILCs $\left(\mathrm{CD}_{4}{ }^{+}, \mathrm{Lin}^{-}, \mathrm{CD} 127^{+}\right)$and ILC2 $\left(\mathrm{CD} 45^{+}\right.$, Lin, $\left.\mathrm{CD} 127^{+}, \mathrm{CD} 25^{+}\right)$in both mice skin. (E) Percentage of $\mathrm{CD} 45^{+}$cells in the skin of PBS-treated and HOCltreated mice at day 42. (F) Percentage of ILC and (G) ILC2, among lymphoid cells in the skin of PBS-treated and HOCl-treated mice at day 42. (H and I) Percentage of KLRG1 ${ }^{+}$cells among ILC2 in the skin of PBS-treated and HOCl-treated mice at day 42 . (J) Positive correlation between the quantity of collagen $(\mu \mathrm{g})$ and the percentage of KLRG1 ${ }^{-}$ILC2. Bar graphs show data as the mean \pm SEM ( $n=10$ per groups). Comparisons between groups were calculated using Mann-Whitney U test. ${ }^{* *} \mathrm{P}<0.01 ;{ }^{* *} \mathrm{p}<0.001 ;{ }^{* * *} \mathrm{p}<0.0001$.

change $=-3.71 ;$ p adjusted value $\left.=1.36 \mathrm{E}^{-51}\right)$, in agreement with the low IL10 expression at the protein level (figure 4C). Transcriptomic results for IL10 and LTC4S were confirmed by Q-RT-PCR (online supplemental figure 4B).

\section{KLRG1- ILC2 are enriched in the skin of hypochlorous acid- treated mice and their numbers correlate with the extent of skin fibrosis}

To assess the in vivo relevance of our findings, we monitored ILC2 in the hypochlorous acid ( $\mathrm{HOCl})$-induced mouse model of SSc, which recapitulates the main hallmarks of $\mathrm{SSc}^{22}$ At day 42, parallel to an increase in dermal thickness (figure 6A for a representative histology of the skin, figure $6 \mathrm{~B}, \mathrm{C}$ for skin thickness and collagen content, respectively), we observed a significant enrichment of the lymphoid cell infiltrate (figure 6D,E). Remarkably, while the frequency of ILCs was not changed (figure $6 \mathrm{~F}$ ), the frequency of ILC2 cells was more than 5 times greater in $\mathrm{HOCl}$-treated skin mice compared with controls as detected by flow cytometry (figure 6G). In addition, the absolute value of cutaneous ILC2 was significantly higher in $\mathrm{HOCl}$ treated mice compared with control mouse (online supplemental figure 5A). This ILC2 enrichment was specific, since the frequency and absolute count of total ILCs was not different in HOCl-treated and non-treated mice (figure $6 \mathrm{~F}$ and online supplemental figure $5 \mathrm{~B}$ ). A slighter increase in ILC2 number and absolute count was already observed even before complete establishment of skin fibrosis (online supplemental figure 5C,D and E,F for skin thickness and collagen content, respectively). Furthermore, the frequency of KLRG1 in ILC2 was fourfold lower in HOCl-treated compared with control mice (figure $6 \mathrm{H}$ for a representative staining of ILC2 and figure 6I for quantification). Interestingly, we observed a strong positive correlation between the proportion of KLRG1 ${ }^{-}$ILC2 2 and the extent of skin fibrosis (figure 6J).

\section{The combined administration of IL10 and TGF $\beta$ inhibition is required to restore skin $\mathrm{KLRG} 1^{+}$ILC2 and to prevent skin fibrosis}

We have shown that the TGF $\beta$-priming of human KLRG1 ILC2 enhances their profibrotic potential by specifically reducing their production of IL10, thus attenuating a negative feedback loop. We then addressed the question of the in vivo relevance of these findings by taking advantage of the $\mathrm{HOCl}$ mouse model of SSc. To inhibit TGF $\beta$ signalling, HOCl-treated mice received daily oral pirfenidone for 42 days, or PBS as control. In addition, they were injected or not with daily IL10. At day 42, the treatment with pirfenidone alone moderately reduced, without reaching statistical significance, the fibrotic response assessed as skin thickness and collagen content (figure 7A,B). The treatment with IL10 by itself had no effect on skin fibrosis. In contrast, when 
A

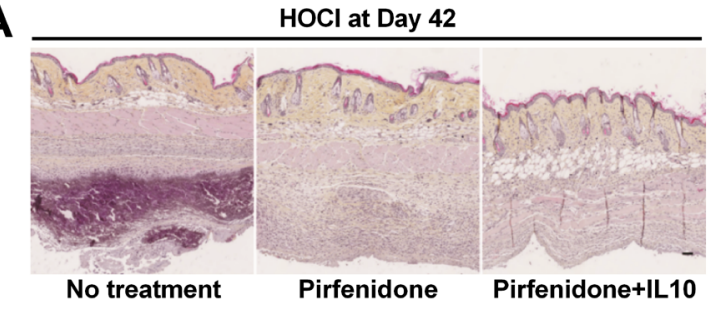

B
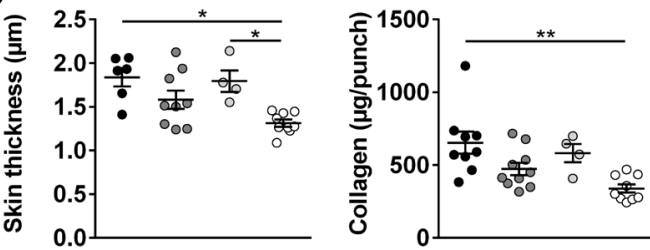

- $\mathrm{HOCl}$

- $\mathrm{HOCl}+$ Pirf

- HOCl+IL10

HOCI+Pirf+IL10

C
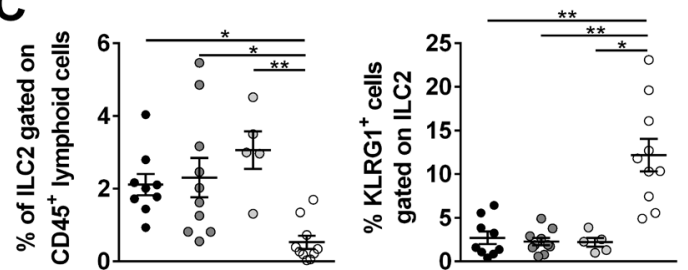

D

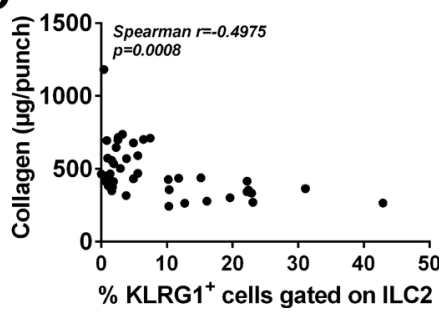

Figure 7 Interleukin (IL)10 and pirfenidone (Pirf) reduce fibrosis and affect the number of innate lymphoid cells (ILC)-2 (ILC2) in the skin of hypochlorous acid ( $\mathrm{HOCl}$ )-treated mice at day 42. (A) H\&E saffron staining of $\mathrm{HOCl}$ skin mice treated or untreated (scale bar $=10 \mu \mathrm{m}$ ). (B) Measure of skin thickness $(\mu \mathrm{m})$ and collagen $(\mu \mathrm{g})$ in the skin of $\mathrm{HOCl}-$ untreated and $\mathrm{HOCl}$-treated mice at day 42. (C) Percentage of ILC2 among lymphoid cells and percentage of $\mathrm{KLRG}^{+}{ }^{+}$cells among ILC2 in the skin of $\mathrm{HOCl}$-untreated and $\mathrm{HOCl}$-treated mice at day 42. (D) Negative correlation between collagen assay $(\mu \mathrm{g})$ and the percentage of $\mathrm{KLRG1}^{+}$ILC2 in the skin. Bar graphs show data as the mean \pm SEM ( $n=10$ per groups). Comparisons between groups were calculated using Kruskal-Wallis test. ${ }^{*} \mathrm{P}<0.05 ;{ }^{*} \mathrm{p}<0.01$.

pirfenidone and IL10 were administered jointly we observed a dramatic and statistically significant reduction in both the skin thickness and collagen content (figure 7B). Interestingly, the proportion of ILC2 was significantly lower and the frequency of their $\mathrm{KLRG}^{+}$subset was significantly higher in $\mathrm{HOCl}$ mice that received the combined treatment with pirfenidone and IL10 when compared with all the other treatments (figure 7C). Not the least, the extent of collagen content in the skin was inversely correlated with the frequency of $\mathrm{KLRG}^{+}$ILC2 (figure 7D). Altogether, these results demonstrate that the combined treatment with pirfenidone and IL10 strongly reduces skin fibrosis in vivo simultaneously reducing the number of ILC2 infiltrating the skin and enhancing their expression of KLRG1.

\section{DISCUSSION}

ILCs were recently identified as new important actors of the innate arm of the immune system. ILCs have been characterised and classified in recent years, and they have been directly implicated in many inflammatory conditions, including fibrosis, atopic dermatitis, asthma and inflammatory bowel disease. ${ }^{23-26}$ SSc is a complex autoimmune connective tissue disease characterised by autoimmunity, widespread tissue fibrosis of the skin and internal organs and vasculopathic alterations. Its pathogenesis remains poorly understood, and a single treatment has not been approved over the last 50 years.

Our study directly implicates the impairment of ILC homeostasis as a potential contributor and new therapeutic strategy for SSc. Specifically, we observed that human skin biopsies from patients with SSc were characterised by a higher proportion of KLRG1 ${ }^{-}$ILC2 positively correlated to the severity of the fibrotic process. Mechanistically, we demonstrated in vitro that TGF $\beta$ decreased KLRG1 expression on ILC2 and enhanced their profibrotic function through IL10 downregulation. Interestingly, in a mouse model of SSc recapitulating human findings, TGF $\beta$ inhibition associated with IL10 administration prevented the development of fibrosis while repopulating the skin via KLRG1 ${ }^{+}$ ILC2 in a synergistic manner. Altogether, those results revealed a previously undescribed mechanism in human SSc pathogenesis and paved the way for potential alternative therapeutic strategies based on TGF $\beta$ blocking associated with IL10 administration.

Few previous studies have investigated the role of ILCs in human SSc. One group described an increased number of CD4 ${ }^{+}$ group 1 ILCs, ${ }^{27}$ while another group reported an increased proportion of ILC2 in the peripheral blood. ${ }^{19}$ In contrast, our data showed a decrease in ILC2 numbers/proportions at the blood level and did not identify a difference for ILC1. We cannot exclude that immunomodulatory agents may have participated to the decrease of ILC2 in the blood. However, in the limit of the number of analysed patients, no impact on the increase amount of ILC2 in the skin of patients with SSc was observed. These contradictory observations also raised specific questions about the phenotypic definition of low-number circulating cells. Although key researchers in the field have reviewed this important question, ${ }^{28}$ differences in gating strategies, antibodies or even methods may explain these discrepancies. In our gating strategy, one may argue that the gate for ILC is too close from the lineage positive cells. As shown in the online supplemental figure $1 \mathrm{~B}$, we pay attention to evaluate that the population edging the lineage positive cells also contained ILC1, ILC2 and ILC3 subsets. Regarding selected markers in our experiments, we used the CD5 in the lineage marker to remove $\mathrm{CD} 4^{+} \mathrm{T}$ lymphocytes. A recent paper showed that $\mathrm{CD}^{+}{ }^{+}$ILCs are functionally immature and very close to progenitor cells. ${ }^{29}$ Although CD5 could be expressed in a small proportion of ILC1, ILC2 and ILC3, these cells become functionally active cytokine-secreting ILCs when they downregulate CD5 and migrate to tissues. Therefore, using CD5 as we have done, appears to be a reasonable trade-off for eliminating CD4 T cells without losing mature cells. Finally, by using two complementary strategies, we observed an increase in the proportion of ILC2 in the SSc skin in accordance with a previously published report. ${ }^{19}$

Extensive characterisation of human skin-homing ILC2 from patients with SSc revealed subtle phenotypic changes associated with the fibrotic process. SSc skin is populated by KLRG1- ILC2, whereas skin-resident ILC2 express KLRG1 in normal skin from humans and mice. Interestingly, KLRG1 expression levels have been described as a marker to separate iILC2 $\left(\mathrm{KLRG}^{+}{ }^{+}\right.$) and nILC2 (KLRG1 $\left.{ }^{-}\right)$. iILC2 could be transient progenitors and develop into nILC2 or ILC3-like cells. ${ }^{30}$ Although this dichotomy has mainly been described in the field of infection in mice, our results show that this observation is 
true in humans and would be directly associated with the fibrotic process. Our results in SSc suggest KLRG1 ${ }^{+}$ILC2 could migrate from the blood to the skin and switch into profibrotic KLRG1 ILC2. Functional studies in vitro revealed that TGF $\beta$, which is known to be elevated in patients with SSc, was a key cytokine involved in KLRG1 downregulation, thus mirroring the effect of TGFb on KLRG1-expressing CD8 ${ }^{+} \mathrm{T}$ cells. ${ }^{31}$ Unexpectedly, we observed that KLRG1- ILC2 triggered profibrotic responses on dermal fibroblast, which was mainly due to an imbalance in production between profibrotic factors and antifibrotic factors. Moreover, we observed that the low capability to secrete IL10 was directly responsible for the profibrotic effect. The role of IL10 as an antifibrotic agent has been previously demonstrated. Indeed, two distinct models of fibrosis, one in chronic renal insufficiency and another in hepatic damage induced by biliary duct ligation, provide evidence for the impact of decreased levels of IL10 in the occurrence and maintenance of fibrosis. ${ }^{32} 33$ Moreover, the genetic delivery of IL10 significantly attenuated TGF $\beta$ production in the lungs of mice subjected to bleomycin-induced pulmonary fibrosis. This effect was still observed when IL10 was delivered at later time points when fibrosis was already established. ${ }^{34}$ Therefore, our results established a direct link between TGF $\beta$ and low secretion of IL10 at the tissue level through ILC2.

To look further on the fibrotic potential of ILC2, we evaluated the transcriptome of TGF $\beta$-stimulated ILC 2 compared with unstimulated ILC2 and found an increase of the leukotriene C4 synthase expression, suggesting that activated ILC2 may release leukotriene C4 (LTC4). Since LTC4 is a potent inducer of collagen synthesis by dermal fibroblasts, ${ }^{35}$ the ILC2-LTC4 axis in SSc skin fibrosis will require further investigations.

Different SSc mice models have been used to evaluate different therapeutic strategies; however, none of them recapitulates the human observations in integro. The HOCl-treated mouse model has the advantage of summarising the three main characteristics of SSc: vasculopathy, deregulation of the immune response (including the production of autoantibodies) and cutaneous and pulmonary fibrosis. In our study, we only tested our hypothesis in one mouse model that constitutes a limitation. However, our data indicated that this model recapitulated the human skin findings, including the increased proportion of KLRG1 ${ }^{-}$skin ILC2 proportions correlated to the fibrotic process. We were not able to increase lung fibrosis, precluding any further pulmonary investigation. Our work is thus limited to only one mouse model exploring cutaneous fibrosis. Many mouse models potentially useful to further assess the relevance of ILC in experimental animals could be considered, of which none fully recapitulates the features of human systemic sclerosis. ${ }^{36}$ However, we believe that, within its limits, the $\mathrm{HOCl}$ mouse model we adopted, strongly support the results of our research principally and extensively made in humans. In detail, it met several important requirements for our demonstration: (i) development of skin fibrosis after an inflammatory period; (ii) accompanied by an increase of ILC2 in the skin; (iii) a positive correlation between skin fibrosis and number of ILC2 in the skin, as we have shown in humans. From a therapeutic perspective, most of the mouse models have implicated ILC2 through its deletion using Rora ${ }^{\mathrm{sg} / \text { flox }} I l 7 r^{\mathrm{Cre} /+}$ mice $^{37}$ or Rag $1^{-/}$mice, for instance, coupled with the injection of depleting anti-Thy1 antibody. ${ }^{38}$ Although these strategies offer a clear view of the global implication of ILC2 in a model, it is not a feasible strategy in humans and does not permit the characterisation of the implication of ILC2-skewed functionalities in a disease process, as it is the case in our setting. Therefore, we decided to adopt an alternative strategy that combines a well-accepted TGF $\beta$ inhibitor and IL10 supplementation, according to our in vitro data. Our results revealed a dramatic synergic effect on the fibrotic process while $\mathrm{KLGR}^{+}{ }^{+}$ILC2 were repopulating the skin. Although this therapeutic strategy does not formally directly prove the involvement of the KLRG1 population in the pathogenesis, it provides indirect evidence on the pathogenic loop implicating ILC2, IL10 and TGF $\beta$. Moreover, our results could constitute the groundwork for proposing a clinical trial testing the combination of the TGF $\beta$ inhibitor and IL10 in patients with SSc. The US Food and Drug Administration recently approved targeting the TGF $\beta$ pathway for the treatment of idiopathic pulmonary fibrosis (IPF). In a phase III clinical trial, pirfenidone successfully reduced the progression of IPF and was associated with fewer deaths. ${ }^{39}$ For unknown reasons, this effect in the patients with SSc seemed to be milder; thus, combining another strategy in this multifactorial disease could be an option. After the successful preclinical experiments, clinical studies using human recombinant IL10 are already in progress for the treatment of inflammatory bowel diseases with an acceptable safety profile. ${ }^{40}$ Future studies using nintedanib instead of pirfenidone are also of interest since this molecule is used in interstitial lung disease in SSc. ${ }^{41}$

In conclusion, our study revealed that ILC2 may contribute to the fibrotic process observed in human SSc and the combination of the TGF $\beta$ inhibitor and IL10 could be a promising therapeutic strategy.

\author{
Author affiliations \\ ${ }^{1}$ ImmunoConcEpt, CNRS, UMR 5164, University of Bordeaux, Talence, France \\ ${ }^{2}$ Rheumatology Department, CHU de Bordeaux, Bordeaux, France \\ ${ }^{3}$ Immunology Department, CHU Cochin Hospital, University of Paris Descartes Faculty \\ of Medicine Paris Center, Paris, France \\ ${ }^{4}$ Animal Facility A2, University of Bordeaux, Talence, France \\ ${ }^{5}$ Centre de Bioinformatique de Bordeaux (CBiB), University of Bordeaux, Talence, \\ France \\ ${ }^{6}$ IBGC, CNRS, UMR 5095, University of Bordeaux, Talence, France \\ ${ }^{7}$ Dermatology Department, CHU de Bordeaux, Bordeaux, France \\ ${ }^{8}$ INSERM U1035, University of Bordeaux, Talence, France \\ ${ }^{9}$ Vascular Medicine Department, CHU de Bordeaux, Bordeaux, France \\ ${ }^{10}$ Immunology and Allergy, University of Geneva, Geneva, Switzerland \\ ${ }^{11}$ Internal Medicine, CHU de Bordeaux, Bordeaux, France \\ ${ }^{12}$ Hematology, CHU de Bordeaux, Bordeaux, France \\ ${ }^{13}$ Immunology department, CHU de Bordeaux, Bordeaux, France
}

Correction notice This article has been corrected since it published Online First. The funding statement has been updated.

Acknowledgements Some of the data were presented in an abstract at the EULAR annual meeting in 2016. Victor Racine and the QuantaCell society are acknowledged for their help with the analysis of the human imaging data. The histological studies were performed at the Experimental Histopathology Platform, INSERM US 005-CNRS UMS 3427-TBM CORE, a service unit of the CNRS-INSERM and Bordeaux University. The help of Nathalie Dugot-Senant is acknowledged. The microscopy analyses were performed at the Bordeaux Imaging Centre, a service unit of the CNRS-INSERM and Bordeaux University, member of the national infrastructure France Biolmaging supported by the French National Research Agency (ANR-10-INBS-04). The help of Sébastien Marais is acknowledged. We are also grateful to Atika Zouine and Vincent Pitard for their expert assistance in flow cytometry (Plateforme de Cytométrie en Flux, SFR TransBioMed, Université Bordeaux), Xavier Gauthereau for assistance in PCR (Plateforme de Q-PCR SFR TransBioMed, Université Bordeaux) and Benoit Rousseau for help in the animal facility (Animalerie A2, Université Bordeaux). RNAseq experiment were realised with the genom'IC platform (INSERM U1016, Institut Cochin). Part of the computational resources and infrastructure used in present publication were provided by the Bordeaux Bioinformatics Centre ( $\mathrm{CBiB}$ ). Eric Vivier and Frédéric Vély are acknowledged for helping us in the setting of ILC staining. Andreas Ramming and Jörg Distler are acknowledged for providing stimulating discussions on the data.

Collaborators Victor Racine, Nathalie Dugot-Senant, Sébastien Marais, Atika Zouine, Vincent Pitard, Xavier Gauthereau, Benoit Rousseau, Eric Vivier, Frédéric Vély, Andreas Ramming, Jörg Distler.

Contributors PL and BA jointly designed the research, performed the experiments, collected, analysed and interpreted the data, wrote and revised the manuscript. MJ, $\mathrm{PM}, \mathrm{EL}, \mathrm{DL}, \mathrm{AG}$ and $\mathrm{VJ}$ performed and analysed some experiments. EL, PL, JI, MJ and FB performed mice experiments. TS, CC, TP and PB provided intellectual input and edited the manuscript. PM, DL, PH, JS, JC, CR, PD, TS, EL and EF recruited the study participants and provided patients' samples and clinical data. CC-B and M-ET jointly designed and supervised the study and wrote the manuscript. 
Funding This research was supported by grants from the Société Française de Rhumatologie (SFR), Rhumato-Network. It was also supported by the Association des Sclérodermiques de France (ASF). Paôline Laurent was funded by a PhD grant from the University of Bordeaux and Valerie Jolivel by an interdisciplinary project of the IDEX (Initiative d'excellence) of the University of Bordeaux (PI: T. Pradeu)

Competing interests None declared.

Patient consent for publication Not required.

Ethics approval Ethical approval information: human participants: Patients were included in the context of the VISS (Vasculopathy and Inflammation in Systemic Sclerosis) biomedical research project founded in 2012 and approved by the institutional ethical committee (CPP, 2012-A00081-42, Aquitaine). All participants provided written informed consent before inclusion. Ethical approval information: animals: Comité d'éthique pour l'expérimentation animale de Bordeaux (CE50) (project $\left.n^{\circ} 15544\right)$.

Provenance and peer review Not commissioned; externally peer reviewed. Data availability statement Data are available on reasonable request.

Supplemental material This content has been supplied by the author(s). It has not been vetted by BMJ Publishing Group Limited (BMJ) and may not have been peer-reviewed. Any opinions or recommendations discussed are solely those of the author(s) and are not endorsed by BMJ. BMJ disclaims all liability and responsibility arising from any reliance placed on the content. Where the content includes any translated material, BMJ does not warrant the accuracy and reliability of the translations (including but not limited to local regulations, clinical guidelines, terminology, drug names and drug dosages), and is not responsible for any error and/or omissions arising from translation and adaptation or otherwise.

Open access This is an open access article distributed in accordance with the Creative Commons Attribution Non Commercial (CC BY-NC 4.0) license, which permits others to distribute, remix, adapt, build upon this work non-commercially, and license their derivative works on different terms, provided the original work is properly cited, appropriate credit is given, any changes made indicated, and the use is non-commercial. See: http://creativecommons.org/licenses/by-nc/4.0/.

\section{ORCID iDs}

Benoit Allard http://orcid.org/0000-0002-7093-5851

Damien Leleu http://orcid.org/0000-0003-3265-3201

Carlo Chizzolini http://orcid.org/0000-0003-4849-6335

Christophe Richez http://orcid.org/0000-0002-3029-8739

Cécile Contin-Bordes http://orcid.org/0000-0003-4652-2907

Marie-Elise Truchetet http://orcid.org/0000-0001-8045-0180

\section{REFERENCES}

1 Denton CP, Khanna D. Systemic sclerosis. Lancet 2017;390:1685-99.

2 Chizzolini C, Brembilla NC, Montanari E, et al. Fibrosis and immune dysregulation in systemic sclerosis. Autoimmun Rev 2011:10:276-81.

3 Laurent P, Sisirak V, Lazaro E, et al. Innate immunity in systemic sclerosis fibrosis: recent advances. Front Immunol 2018;9:9.

4 Mahoney JM, Taroni J, Martyanov V, et al. Systems level analysis of systemic sclerosis shows a network of immune and profibrotic pathways connected with genetic polymorphisms. PLoS Comput Biol 2015;11:e1004005.

5 Klose CSN, Artis D. Innate lymphoid cells as regulators of immunity, inflammation and tissue homeostasis. Nat Immunol 2016;17:765-74.

6 Spits $H_{1}$ Artis D, Colonna M, et al. Innate lymphoid cells--a proposal for uniform nomenclature. Nat Rev Immunol 2013;13:145-9.

7 Constantinides MG, McDonald BD, Verhoef PA, et al. A committed precursor to innate lymphoid cells. Nature 2014;508:397-401.

8 Jia Y, Fang X, Zhu X, et al. IL-13 ${ }^{+}$Type 2 Innate Lymphoid Cells Correlate with Asthma Control Status and Treatment Response. Am J Respir Cell Mol Biol 2016;55:675-83.

9 Smith SG, Chen R, Kjarsgaard M, et al. Increased numbers of activated group 2 innate lymphoid cells in the airways of patients with severe asthma and persistent airway eosinophilia. J Allergy Clin Immunol 2016;137:75-86.

10 Meng X-M, Nikolic-Paterson DJ, Lan HY. TGF- $\beta$ : the master regulator of fibrosis. Nat Rev Nephrol 2016;12:325-38.

11 Lafyatis R. Transforming growth factor $\beta$--at the centre of systemic sclerosis. Nat Rev Rheumatol 2014;10:706-19.

12 Varga J, Whitfield ML. Transforming growth factor-beta in systemic sclerosis (scleroderma). Front Biosci 2009;1:226-35.

13 Wang L, Tang J, Yang X, et al. TGF- $\beta$ induces ST2 and programs ILC2 development. Nat Commun 2020;11:1-15.
14 Huang Y, Guo L, Qiu J, et al. IL-25-responsive, lineage-negative KLRG1(hi) cells are multipotential 'inflammatory' type 2 innate lymphoid cells. Nat Immunol 2015;16:161-9.

15 Huang $Y$, Mao K, Chen X, et al. S1P-dependent interorgan trafficking of group 2 innate lymphoid cells supports host defense. Science 2018;359:114-9.

16 Lonati PA, Brembilla NC, Montanari E, et al. High IL-17E and low IL-17C dermal expression identifies a fibrosis-specific motif common to morphea and systemic sclerosis. PLoS One 2014:9:e105008.

17 Manetti M, Guiducci S, Ceccarelli C, et al. Increased circulating levels of interleukin 33 in systemic sclerosis correlate with early disease stage and microvascular involvement. Ann Rheum Dis 2011;70:1876-8.

18 Truchetet M-E, Demoures B, Eduardo Guimaraes J, et al. Platelets induce thymic stromal lymphopoietin production by endothelial cells: contribution to fibrosis in human systemic sclerosis. Arthritis Rheumatol 2016;68:2784-94.

19 Wohlfahrt T, Usherenko S, Englbrecht M, et al. Type 2 innate lymphoid cell counts are increased in patients with systemic sclerosis and correlate with the extent of fibrosis. Ann Rheum Dis 2016;75:623-6.

20 Ohne Y, Silver JS, Thompson-Snipes L, et al. II-1 is a critical regulator of group 2 innate lymphoid cell function and plasticity. Nat Immunol 2016;17:646-55.

21 Yagi R, Zhong C, Northrup DL, et al. The transcription factor GATA3 is critical for the development of all IL-7R $\alpha$-expressing innate lymphoid cells. Immunity 2014; $40: 378-88$

22 Servettaz A, Goulvestre C, Kavian N, et al. Selective oxidation of DNA topoisomerase 1 induces systemic sclerosis in the mouse. J Immunol 2009;182:5855-64.

23 Geremia A, Arancibia-Cárcamo CV, Fleming MPP, et al. IL-23-responsive innate lymphoid cells are increased in inflammatory bowel disease. J Exp Med 2011;208:1127-33.

24 Hams E, Armstrong ME, Barlow JL, et al. II-25 and type 2 innate lymphoid cells induce pulmonary fibrosis. Proc Natl Acad Sci U S A 2014;111:367-72.

25 Jia Y, Fang X, Zhu X, et al. IL-13+ Type 2 Innate Lymphoid Cells Correlate with Asthma Control Status and Treatment Response. Am J Respir Cell Mol Biol 2016;55:675-83.

26 Salimi M, Barlow JL, Saunders SP, et al. A role for IL-25 and IL-33-driven type-2 innate lymphoid cells in atopic dermatitis. J Exp Med 2013;210:2939-50.

27 Roan F, Stoklasek TA, Whalen E, et al. Cd4+ group 1 innate lymphoid cells (ILC) form a functionally distinct ILC subset that is increased in systemic sclerosis. J Immunol 2016;196:2051-62.

28 Vallentin B, Barlogis V, Piperoglou C, et al. Innate lymphoid cells in cancer. Cancer Immunol Res 2015;3:1109-14.

29 Nagasawa M, Germar K, Blom B, et al. Human $\mathrm{CD}^{+}$Innate Lymphoid Cells Are Functionally Immature and Their Development from CD34+ Progenitor Cells is Regulated by Id2. Front Immunol 2017;8:1047.

30 Huang Y, Paul WE. Inflammatory group 2 innate lymphoid cells. Int Immunol 2016:28:23-8.

31 Schwartzkopff S, Woyciechowski S, Aichele U, et al. TGF- $\beta$ downregulates KLRG1 expression in mouse and human CD8(+) T cells. Eur J Immunol 2015;45:2212-7.

32 Mu W, Ouyang X, Agarwal A, et al. II-10 suppresses chemokines, inflammation, and fibrosis in a model of chronic renal disease. J Am Soc Nephrol 2005;16:3651-60.

33 Sharawy MH, Abdel-Rahman N, Megahed N, et al. Paclitaxel alleviates liver fibrosis induced by bile duct ligation in rats: role of TGF- $\beta 1$, IL-10 and c-myc. Life Sci 2018:211:245-51.

34 Nakagome K, Dohi M, Okunishi K, et al. In vivo IL-10 gene delivery attenuates bleomycin induced pulmonary fibrosis by inhibiting the production and activation of TGF-beta in the lung. Thorax 2006;61:886-94.

35 Oyoshi MK, He R, Kanaoka Y, et al. Eosinophil-derived leukotriene C4 signals via type 2 cysteinyl leukotriene receptor to promote skin fibrosis in a mouse model of atopic dermatitis. Proc Natl Acad Sci U S A 2012;109:4992-7.

36 Marangoni RG, Varga J, Tourtellotte WG. Animal models of scleroderma: recent progress. Curr Opin Rheumatol 2016;28:561-70.

37 Oliphant CJ, Hwang YY, Walker JA, et al. MHCII-mediated dialog between group 2 innate lymphoid cells and CD4(+) T cells potentiates type 2 immunity and promotes parasitic helminth expulsion. Immunity 2014;41:283-95.

38 Monticelli LA, Sonnenberg GF, Abt MC, et al. Innate lymphoid cells promote lung-tissue homeostasis after infection with influenza virus. Nat Immunol 2011:12:1045-54.

39 Azuma A, Taguchi Y, Ogura T, et al. Exploratory analysis of a phase III trial of pirfenidone identifies a subpopulation of patients with idiopathic pulmonary fibrosis as benefiting from treatment. Respir Res 2011;12:143.

40 Fedorak RN, Gangl A, Elson CO, et al. Recombinant human interleukin 10 in the treatment of patients with mild to moderately active Crohn's disease. the interleukin 10 inflammatory bowel disease Cooperative Study Group. Gastroenterology 2000;119:1473-82

41 Seibold JR, Maher TM, Highland KB, et al. Safety and tolerability of nintedanib in patients with systemic sclerosis-associated interstitial lung disease: data from the SENSCIS trial. Ann Rheum Dis 2020;79:1478-84. 